\title{
Demonstration of Slow Photon Chemistry on Multilayer Inverse Opals
}

\author{
Delphine Misao Lebrun and Lars Österlund* \\ Department of Engineering Sciences, The Ångström Laboratory, Uppsala University, P.O. Box 534, \\ SE-75121 Uppsala, Sweden
}

\begin{abstract}
We demonstrate the use of atomic layer deposited multilayered alumina/titania photonic crystals for stearic acid (SA) degradation using broadband light illumination. The degradation of SA was monitored every 15 minutes by Fourier transform infrared spectroscopy, using a simple rotation of the sample holder to switch between light source and infrared probing beam. We analyzed four samples with different photonic bandgap (PBG) positions, from 397 to $372 \mathrm{~nm}$, with different titania layer thicknesses, but otherwise prepared in identical manner. With this approach the photonic efficiency (PE) could readily be calculated without having to consider different sample preparation procedure. We found that a sample with a high-energy edge of the PBG position overlapping with the electronic bandgap position of titania $\left(E_{\mathrm{g}} \sim 385 \mathrm{~nm}\right)$ and the highest photon power of our light source had the highest $P E=0.002$. Comparison with the other samples shows that the slow photon effect apparent at the PBG edge can explain the higher PE, and suggest that bandgap engineering of multilayer photonic materials is a practically viable method to improve the efficiency of a photocatalyst.
\end{abstract}

KEYWORDS: Photocatalysis, Photonic Crystals, Slow Photon, Titania, Stearic Acid.

\section{INTRODUCTION}

Water and air cleaning materials using solar energy have been intensely investigated, ${ }^{1}$ and particularly the use of titanium dioxide $\left(\mathrm{TiO}_{2}\right)$, since the publication of Fujishima and Honda in 1972. ${ }^{2}$ There are several advantages to the use of titania, as it is abundant, non-toxic, and photoresistant. ${ }^{3}$ Unfortunately, it is an indirect electronic band gap material, ${ }^{4}$ and, as such, possess a low electron-hole pair creation rate, which is directly linked to the efficiency of photocatalysis. ${ }^{5}$ To palliate this defect, two routes are possible: ${ }^{6}$ modifying the material (e.g., by dopants or co-catalysts), or use structuring of the material to control the light propagation. To increases the electron-hole pair generation in $\mathrm{TiO}_{2}$, we choose to modify its structure and fabricated photonic crystals. Photonic crystals are structures composed with at least two dielectrics arranged periodically. The periodicity and the type of material will give rise to a photonic band structure. ${ }^{7-9}$ Particularly, if the two materials have a high refractive index contrast, a photonic bandgap (PBG) will be formed. A PBG is a forbidden energy state for the photon within the structure, meaning that incoming light with the PBG energy will be totally reflected. The interest of $3 \mathrm{D}$ photonic crystals

*Author to whom correspondence should be addressed.

Email: lars.osterlund@angstrom.uu.se

Received: 1 December 2015

Accepted: 13 May 2016 in photocatalysis lies in the edges of the PBG. ${ }^{10,11}$ Light with energy close to the PBG but not at the PBG, will experience multiple reflections inside the structure at each interface between the two materials, thereby increasing the effective path length for the light. This effect had been called the slow light effect and has been shown to increase the absorption coefficient, due to a longer effective path length in the $\mathrm{TiO}_{2}$ inverse opals, and therefore increase its photocatalytic efficiency. ${ }^{12,13}$

In this paper, we demonstrate the enfanced photodegradation of stearic acid (SA) on atomic layer deposited (ALD) multilayered alumina $\left(\mathrm{Al}_{2} \mathrm{O}_{3}\right) /$ titania $\left(\mathrm{TiO}_{2}\right)$ inverse opals (3D photonic crystals), using broadband light illumination.

\section{EXPERIMENTAL DETAILS}

\subsection{Chemicals}

Photonic crystal films were fabricated on quartz substrates (TedPella, Redding, USA). Polystyrene spheres (PS) with a diameter of $200 \mathrm{~nm}$ dispersed in water (ThermoScientific, USA) were used to fabricate templates. The PS templates were self-assembled at $50{ }^{\circ} \mathrm{C}$ using a solution concentration of $0.002 \mathrm{~g} / \mathrm{mL}(0.2 \% \mathrm{w} / \mathrm{v})$. Decon90 (Decon Laboratories Limited, UK) was used as received to clean the quartz substrates and all glassware involved. 
Trimethylaluminium (TMA; SAFC Hitech) and titanium tetrachloride $\left(\mathrm{TiCl}_{4}\right.$; Picosun $\left.\mathrm{Oy}\right)$ were used as precursors in ALD for alumina and titania deposition, respectively.

A solution of $0.08 \mathrm{mM}$ SA (Merck Shuchardt OGH, Germany) was dissolved in methanol (VWR SA, France). For all the photocatalytic measurements, $1 \mathrm{~mL}$ of the solution was spin coated (around $2500 \mathrm{rpm}$ ) onto the sample and dried under $\mathrm{N}_{2}$ gas flow (1 bar, $1 \mathrm{~min}$ ) prior to the photodegradation experiments.

\subsection{Inverse Multilayer Opals}

Four different samples were probed with the FTIR setup. All samples were prepared with a $0.2 \mathrm{w} / \mathrm{v} \%$ polystyrene solution in water at $50{ }^{\circ} \mathrm{C}$ in an oven, using the vertical convective evaporation method, with $200 \mathrm{~nm}$ bead diameter and on quartz substrates. All samples were annealed at $85{ }^{\circ} \mathrm{C}$ and around $15 \mathrm{~nm}$ of $\mathrm{Al}_{2} \mathrm{O}_{3}$ were deposited onto the polystyrene opals using ALD. After template removal, around $4 \mathrm{~nm}$ of $\mathrm{TiO}_{2}$ were deposited with ALD onto the samples (A) and (B), while around $2.5 \mathrm{~nm}$ of $\mathrm{TiO}_{2}$ were deposited onto samples (C) and (D). The PS template coverage of the quartz substrates was not uniform, so the samples have a different total area and thickness. In Table I it is seen that samples (A) and (B) have similar PBG, while sample (C) and (D) show a significant variation, which we attribute to varitions of effective refractive index of the composite structure in the $2.5 \mathrm{~nm}$ films due to incomplete $\mathrm{TiO}_{2}$ film formation, and variations in aggregated $\mathrm{TiO}_{2}$ size distribution on the alumina walls during ALD deposition.

\subsection{Materials Characterization}

Optical transmittance and reflectance were obtained using a spectrophotometer (Lambda900, Perkin Elmer, U.S.A.). The optical data were used to assess the template thickness with a method, which is subject for a forthcoming publication, which uses the known absorption coefficient of PS at $262 \mathrm{~nm}$ and the measured optical density. X-ray diffraction (Siemens D5000, Germany, with a radiation $\mathrm{CuK}_{\alpha 1}=$ $1.541 \AA$ ) measurements at grazing angle were perfomed on the multilayer inverse opals: alumina was found to be amourphous, while titania had anatase crystalline phase after post-annealing.

Table I. Photonic band gap (PBG) position of the samples, calculated template film thickness $(t)$ and sample area, calculated SA coverage $\theta$, degradation rate constant and corresponding $\mathrm{PE}$ using a constant total flux of $9.29 \times 10^{14}$ number of photon/ $/ \mathrm{cm}^{2} \mathrm{~s}$, for all samples.

\begin{tabular}{lccrccc}
\hline Name & PBG $(\mathrm{nm})$ & $t(\mu \mathrm{m})$ & $\begin{array}{c}\text { Sample } \\
\text { area }\left(\mathrm{cm}^{2}\right)\end{array}$ & $\theta$ & $k(/ \mathrm{min})$ & PE $\left(\times 10^{3}\right)$ \\
\hline A & 397 & 8.2 & 145.7 & 1.10 & 0.005 & 2.09 \\
B & 396 & 8.3 & 148.9 & 0.51 & 0.0036 & 0.51 \\
C & 385 & 4.5 & 88.0 & 2.89 & 0.0016 & 0.38 \\
D & 372 & 7.3 & 166.2 & 0.93 & 0.0011 & 0.11 \\
\hline
\end{tabular}

\subsection{Photocatalysis Setup}

Figure 1 shows the setup used for photocatalytic measurements. To measure SA concentration, we used Fourier Transform Infra-Red (FTIR) spectroscopy (IFS 66 v/S, Bruker, Germany) combined with a $200 \mathrm{~W} \mathrm{Hg}$ (Xe) arc lamp (Spectra-Physics, U.S.A.) as light source, equipped with AM0 and AM1.5 filters (Thermo Oriel, U.S.A.). The sample holder was situated inside the open sample compartiment mounted on a manual in-plane rotation stage. A central round aperture of $1.3 \mathrm{~cm}$ diameter was used as probe area for the IR signal. A reflecting gold mirror was at an angle towards the light source, at around $15 \mathrm{~cm}$ from the sample holder, to reflect light onto the sample. The normal incidence of the IR beam corresponded to a position of $64^{\circ}$ on our sample holder, and the normal light incidence was set at $102^{\circ}$, so that the sample was rotated $38^{\circ}$ between illumination time and measurement time. The background spectrum was taken after SA loading and prior to illumination, from 4000 to $600 \mathrm{~cm}^{-1}$. Spectra were then taken every 15 minutes after the illumination of the sample.

We recorded 120 times the interferrogram with a resolution of $4 \mathrm{~cm}^{-1}$, using an aperture of $1 \mathrm{~mm}$. The scan time for both background and measured spectra was $1 \mathrm{~min}$.

The total distance between sample holder and light source was $1.1 \mathrm{~m}$. Photon flux (number of photons $/ \mathrm{cm}^{2} \mathrm{~s}$ ) was determined from the manufacturer irradiance data $\left(\mathrm{W} / \mathrm{cm}^{2} \mathrm{~nm}\right)$ and the photon energy between 200 to $400 \mathrm{~nm}$; corrected for the use of filters and the loss of light by the gold mirror. We measured a power of around $0.122 \mathrm{~W} / \mathrm{cm}^{2}$ at the place of the sample holder using a thermopile detector (Bfi Optilas $\mathrm{GmbH}$, Germany). The total output power of the lamp $\left(P_{l}\right)$ was calibrated using the tabulated irradiance of a naked $\mathrm{Hg}$ lamp $(I)$, corrected with the filters transmission $\left(T_{\mathrm{AM}}\right)$ and gold mirror reflection $\left(R_{\mathrm{Au}}\right)$, following:

$$
P_{l}=\int T_{\mathrm{AM}} \times R_{\mathrm{Au}} \times I d \lambda
$$

A conversion factor $(f)$ between tabulated $\left(P_{l}\right)$ and measured $\left(P_{m}\right)$ power can be established by:

$$
P_{m}=f \times P_{l}
$$

The conversion factor $(f=100.02)$, was then applied on the irradiance data from the wavelength range 200 to $400 \mathrm{~nm}$.

The reflectance $\left(R_{\mathrm{T}}\right)$ of the Au mirror was determined by spectrophotometry from the measured diffused $\left(S_{\mathrm{D}}\right)$ and total reflection $\left(S_{\mathrm{T}}\right)$ signals, and calculated using the following equations:

$$
R_{\mathrm{T}}=0.96\left(S_{\mathrm{T}}-S_{\mathrm{D}}\right)+S_{\text {ref }} \cdot S_{\mathrm{D}}
$$

Where $S_{\text {ref }}$ the signal of the Lambda900 setup and 0.96 is a correction factor inherent to the instrument. 


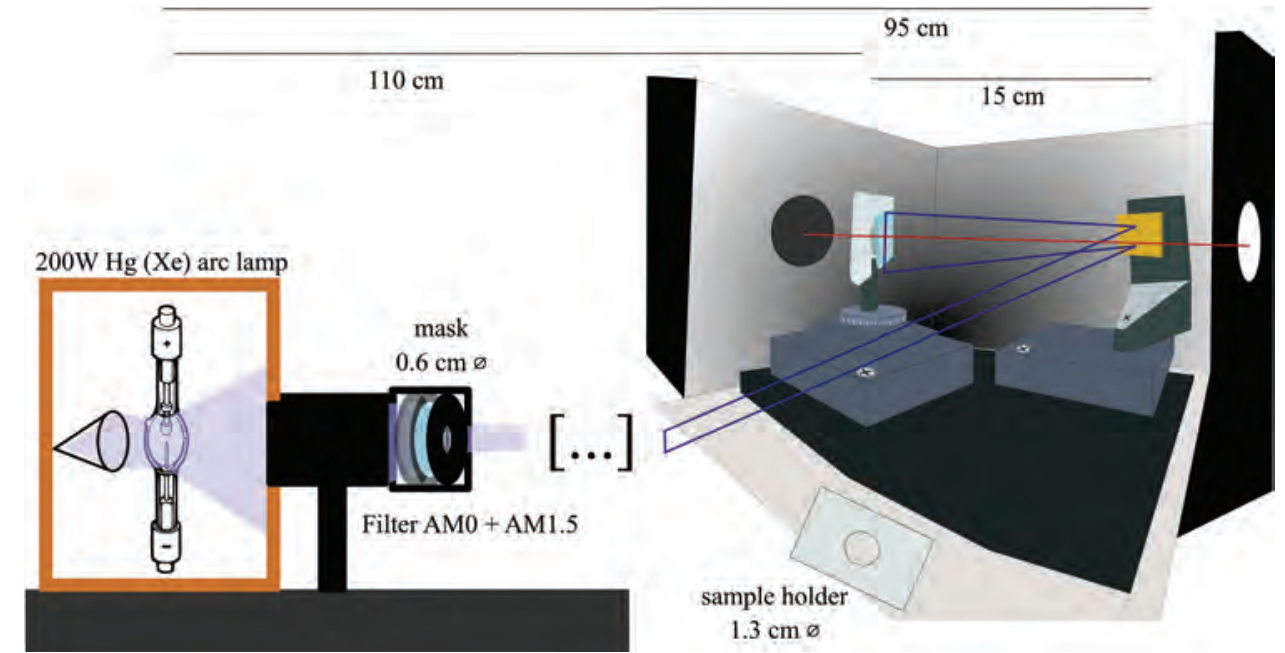

Fig. 1. FTIR setup schematics: Arc lamp with filters (left) positioned $95 \mathrm{~cm}$ away from a gold mirror, placed inside the FTIR reaction chamber (right). The sample holder is situated $15 \mathrm{~cm}$ from the mirror. A spare sample holder can be seen on the bottom right. The blue lines represent the visible light path and the red line represents the IR beam path.

Similarly, the total transmission $\left(T_{\mathrm{T}}\right)$ of the filters was calculated from diffuse $\left(S_{\mathrm{D}}\right)$ and total $\left(S_{\mathrm{T}}\right)$ transmission measurements, using the following equations:

$$
T_{\mathrm{T}}=S_{\mathrm{T}}-S_{\mathrm{D}}+S_{\text {ref }} \cdot S_{\mathrm{D}}
$$

This procedure allows the photon flux per nanometer (number of photons $/ \mathrm{cm}^{2} \mathrm{~s} \mathrm{~nm}$ ) to be determined. We divided the calculated irradiance by the corresponding photon energy, and integrated the values in the wavelength range 200 to $400 \mathrm{~nm}$. At the surface of our samples, the total flux was calculated to be around $9.29 \times 10^{14}$ number of photons $/ \mathrm{cm}^{2} \mathrm{~s}$.

\section{RESULTS AND DISCUSSION}

\subsection{Stearic Acid Photodegradation}

The IR beam probes the $v(\mathrm{C}-\mathrm{H})$ stretching bonds of stearic acid $^{14}$ (SA) between $2700 \mathrm{~cm}^{-1}$ and $3000 \mathrm{~cm}^{-1}$. Two strong vibrational responses were observed at $2916 \mathrm{~cm}^{-1}$ and $2850 \mathrm{~cm}^{-1}$, corresponding to the asymmetric and symmetric $v(\mathrm{C}-\mathrm{H})$ stretching of the $\mathrm{CH}_{2}$ bond respectively. A third, smaller, vibrational response was observed around $2958 \mathrm{~cm}^{-1}$, corresponding to the asymmetric $v(\mathrm{C}-\mathrm{H})$ stretching of the $\mathrm{CH}_{3}$ bond. ${ }^{15}$

Figure 2 shows the measured IR transmittance versus wavenumber for sample A, with a spectrum taken every 15 minutes. Transmission of the bare sample was used to obtain the absorption spectra. To obtain the number of molecules degraded per time, we calculated the total area of the IR peaks, after baseline correction, from $2700 \mathrm{~cm}^{-1}$ to $3000 \mathrm{~cm}^{-1}$. The reported conversion value ${ }^{16}$ between FTIR absorbance in the 2700 to $3000 \mathrm{~cm}^{-1}$ region and the number of SA molecules per $\mathrm{cm}^{2}$ can be used to can then be used to convert the absorbance, $A=-\log _{10}(T)$, from the measured transmittance $(T)$ spectra to number of molecules: $c v=9.7 \times 10^{15} \mathrm{SA}$ molecules $\mathrm{cm}^{-2} \equiv 1 \mathrm{~cm}^{-1}$. From the calculated area at each illumination time (every $15 \mathrm{~min}$ ), and assuming a first-order kinetics, it is possible to determine the degradation rate constant $k$, using:

$$
-\frac{d A}{d t}=k A
$$

with $A$ as the integrated absorbance unit per $\mathrm{cm}^{-1}$, corresponding to SA concentration $C$, and $k$ per $\min ^{-1}$. The plot of the logarithm of the normalized integrated absorbance (area in the range $2700 \mathrm{~cm}^{-1}$ to $3000 \mathrm{~cm}^{-1}$ ) versus time should give a straight line, with a slope value of $k$.

Figure 3 shows the plotted logarithm of $C / C_{0}$ versus illumination time ( $C_{0}$ is the initial concentration), where the linear fit directly provides a value for the degradation rate constant $k$. The total number of absorbed SA can be derived from the area of the absorbance, using the conversion value $c v$. The maximum number of SA monolayers on the sample can then be calculated, using the area of one

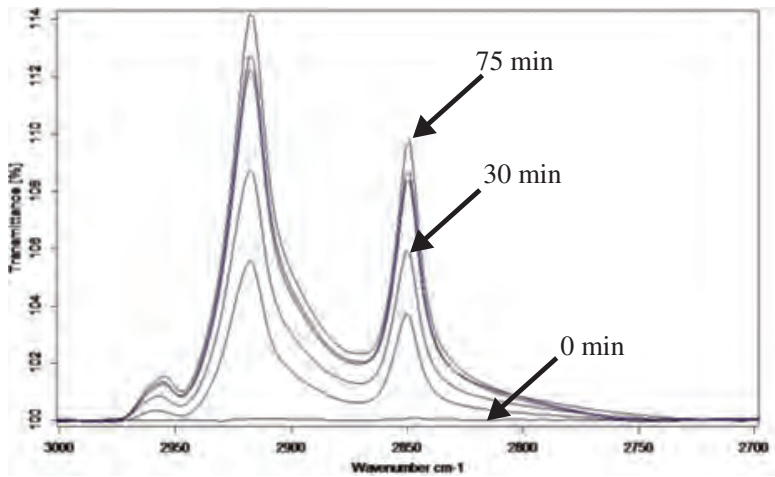

Fig. 2. FTIR transmittance measurements of sample $A$, the background is displayed with a dark vertical line and the successive FTIR spectra in dark blue, every 15 minutes of illumination. 


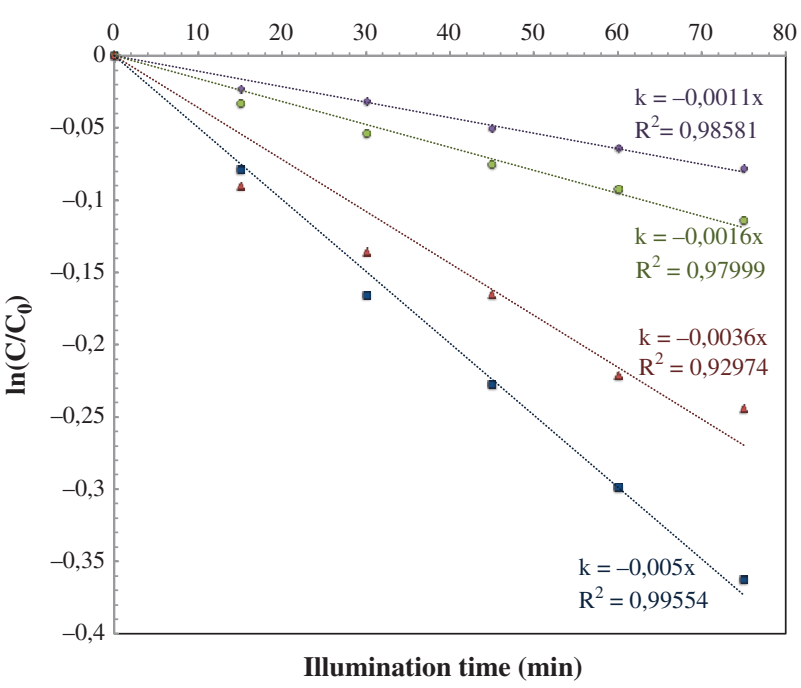

Fig. 3. Logarithm of $C / C_{0}$ versus illumination time. Sample (A) in blue $\square$, (B) in red $\triangle$, (C) in green $\bigcirc$ and (D) in purple $\diamond$, with the corresponding linear fit (dotted lines) and slope value (degradation rate constant $k$ ) with $R$-squared value. Peak area evaluated with a \pm 0.02 error.

molecule of $\mathrm{SA}^{17}\left(0.21 \mathrm{~nm}^{2}\right)$ and the theoretical surface area of the samples (calculated from thickness, substrate area and number of layers in a fcc stacking). This define the coverage $\theta$ of the samples by: ${ }^{18}$

$$
\theta=\frac{\text { Number of SA on the surface }}{\text { Maximum number of SA on the surface }}
$$

The SA coverage $\theta$ at $t=0$ are shown in Table I, samples (A) and (C) have more than a monolayer coverage, while sample (B) and (D) has less than one layer of SA.

We can see that samples (A), (B) and (D) have similar surface area and that sample (C) has the lowest, however, sample (C) has the highest coverage (almost 3 SA layers). Sample (A) and (D) have similar coverage, while sample (B) has less than a monolayer. It shows ${ }^{19}$ that samples (A) and (D) have more active sites than sample (B). For sample $(\mathrm{C})$, the coverage is around three times that of the coverage of the other samples. The consequences of a higher SA concentration is not straightforward, the multi-layer could interfere with products desorption, ${ }^{20}$ thus lowering the otherall degradation of SA; and the first layer can interfere between the surface of the catalyst and the second layer.

Table I shows the calculated degradation rate and photonic efficiency $(P E),{ }^{16,23}$ as defined by:

$$
\mathrm{PE}(\mathrm{SA})=\frac{k(1 / \mathrm{s}) \times \Delta \theta \times c v}{\text { photon flux (photons } \left./ \mathrm{cm}^{2} \mathrm{~s}\right)}
$$

with $\Delta \theta$ the difference in SA coverage between at $t=0$ and $t=75 \mathrm{~min}$ and $c v$ in SA molecules $/ \mathrm{cm}^{2}$. As it can be seen, sample $(\mathrm{A})$ is the best performing sample ( $\mathrm{PE}=$ 0.002), followed by samples (B), (C) and then (D).

\subsection{Factor Influencing the SA Degradation}

To be able to understand the difference in photocatalytic rates among similarly prepared samples and similar experimental setup, it is necessary to review the characteristics of photonic crystal samples: the position of the PBG and the quality of the PBG effect. The PBG is associated with a total reflection, meaning that light within the bandgap will not penetrate inside the sample and therefore cannot be used for electron-hole pair creation. Although, light with energies in the edges of the PBG will experience multiple reflections inside the material, effectively reducing the group velocity to zero (standing waves). This effect has been denominated slow light. ${ }^{24}$ The better the quality of the photonic crystal, the stronger the effects on the light associated with a PBG. A good parameter to assess the quality of the photonic crystal can be derived from the reflection spectrum. At the PBG, a reflection $\left(R_{\mathrm{PBG}}\right)$ occurs and can be compared to the background $\left(R_{\mathrm{BKG}}\right)$ using:

$$
\Delta R=\frac{R_{\mathrm{PBG}}-R_{\mathrm{BKG}}}{100-R_{\mathrm{BKG}}}
$$

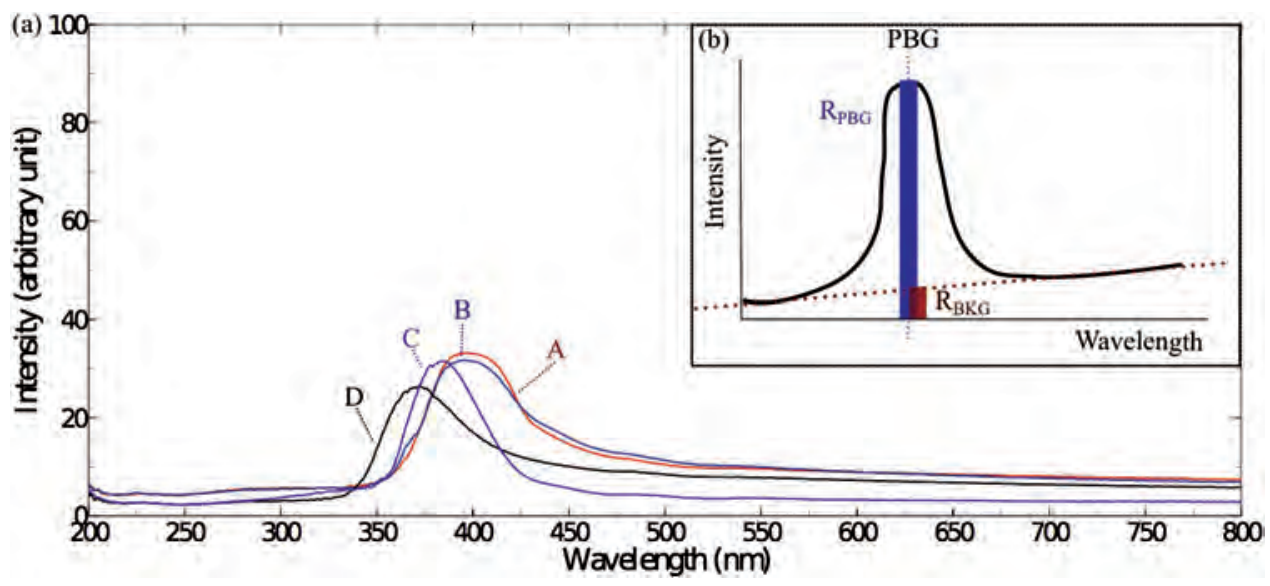

Fig. 4. Total reflection spectra (a) experimental data for sample (A) in red, (B) in blue, (C) in purple and (D) in black, and (b) onset showing how $\mathrm{PBG}, R_{\mathrm{PBG}}$ and $R_{\mathrm{BKG}}$ were measured. 
Table II. Descending ranking of the samples characteristics: calculated surface area (theoretical surface area from sample thickness and inverse opal characteristics), calculated coverage $\theta$, measured PBG position, calculated $\Delta R$ quality factor and calculated PE.

\begin{tabular}{lllll}
\hline Rank & 1 & 2 & 3 & 4 \\
\hline Surface area & D & B & A & C \\
Coverage $\theta$ & C & A & D & B \\
PBG position & A & B & C & D \\
$\Delta R$ & C & A & B & D \\
PE & A & B & C & D \\
\hline
\end{tabular}

where $\Delta R$ represent the normalized total reflection at the PBG.

The better quality (less defects) photonic crystals will have a higher $\Delta R$ (a perfect photonic crystal $\Delta R=1$ ). Figure 4 shows all the total reflection spectra, which allow us to identify the PBG and the quality factor $\Delta R$ of the samples (see Table II). Sample (C) has a better quality factor, followed closely by sample (A), while sample (D) has the lowest $\Delta R$.

\subsection{Slow Photon Chemistry}

To explain the difference in the PE of the different samples, we can use Table II to compare their different characteristics, and Figure 5, explaining the importance of the PBG regarding the material used $\left(\mathrm{TiO}_{2}\right)$ and the type of light source (sun or indoor lights). To use efficiently the edges effect of the PBG, as it can be seen in Figure 5, the PBG (with a band width of around $50 \mathrm{~nm}$, calculated from the optical data) need to be close to the electronic bandgap of $\mathrm{TiO}_{2}$. If the PBG happens to be inside the absorption range of titania, part of the incoming light in this energy range will be reflected, which of course reduce the electron-hole pair creation. The PBG edges need to be near the electronic bandgap edge, to favor photon absorption by $\mathrm{TiO}_{2}$, but also close to the highest lamp irradiance (peak at around $360 \mathrm{~nm}$ ). Samples (A) and (B), as seen

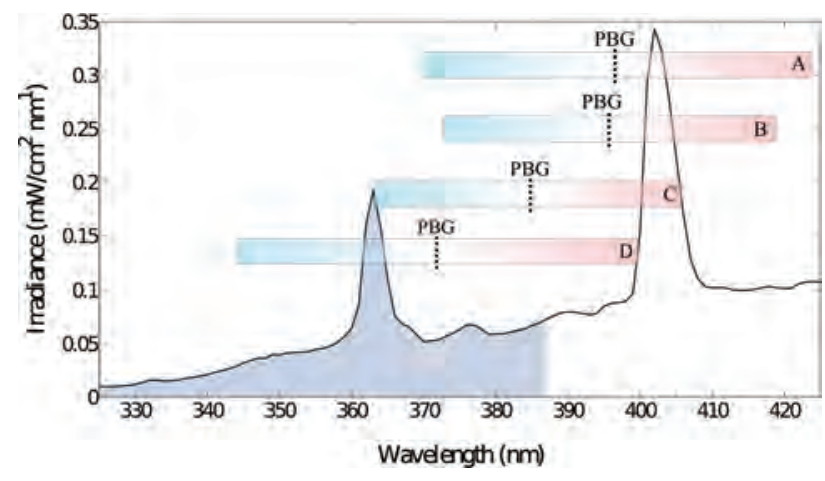

Fig. 5. Schematics of the lamp irradiance (black line) versus wavelength, with the absorption of $\mathrm{TiO}_{2}$ displayed in dark blue and the experimental position of the PBG of samples (A) to (D) with vertical dotted lines. The edges of the PBG are displayed in light blue and red, and the widths of the PBG are extracted from Figure 4. in Figures 4 and 5, have a PBG position close to $390 \mathrm{~nm}$, which is a great position regarding the absorption edge of $\mathrm{TiO}_{2}$ (situated around $385 \mathrm{~nm}^{25}$ ). On the contrary, samples (C) and (D) have a PBG situated within the absorption range of titania, and therefore a competitive effect arises, between loss due to reflected light and gain from increased light absorption at higher energies.

We can now explain easily why sample (A) has the highest PE and sample (B) does not. These samples have a favorable PBG position, where the light reflected by the structure cannot be absorbed, but sample (A) not only has a stronger PBG effect, but also its blue edge at higher energies than sample (B), meaning at the $360 \mathrm{~nm}$ peak. As seen in Figure 5, sample (D) has a PBG reflecting almost all the incoming light with energies below $3.35 \mathrm{eV}$, which correspond also to the highest irradiance of our light source. Sample (C) has a higher PE than sample (D), but lower than sample (A). We know that sample (C) posses the higher PBG quality (Eq. (6)) with the high-energy edge overlapping the $\mathrm{Hg}$ emission peak at around $360 \mathrm{~nm}$, but the PBG of sample (C) is situated within $\mathrm{TiO}_{2}$ absorption energy range and has a much higher coverage, which could explain why sample (A) as a higher PE.

\section{CONCLUSION}

Using FTIR spectroscopy of stearic acid degradation under broadband light illumination, we demonstrated the slow light effect of ALD deposited $\mathrm{Al}_{2} \mathrm{O}_{3} / \mathrm{TiO}_{2}$ 3D photonic crystals. Sample with a photonic band gap $(\mathrm{PBG}=397 \mathrm{~nm})$ close to the electronic bandgap of titania $\left(E_{\mathrm{g}} \sim 385 \mathrm{~nm}\right)$ shows a high photonic efficiency $\left(P E_{397} \mathrm{~nm} \sim 0.002\right)$. The preparation method allows a more refine control on the PBG position and a similarity in sample morphology, which facilitates quantitative comparisons.

These experiments allow us to understand the factors to take into consideration, regarding photonic crystals uses in photocatalysis. It is not only important to engineer the position of the PBG carefully regarding the type of material forming the structure, but also the conditions of illumination and the quality of the photonic crystals, for a better photocatalytic performance.

Acknowledgments: This work was funded by the Swedish Research Council Grant Agreement No. 20103514. The paper was presented at the IUMRS-ICAM 2015 conference, 25th to 29th of October 2015, at the International Conference Center, Jeju-Do, South-Korea.

\section{References and Notes}

1. M. R. Hoffmann, S. T. Martin, W. Choi, and D. W. Bahnemann, Chem. Rev. 95, 69 (1995).

2. A. Fujishima and K. Honda, Nature 238, 37 (1972).

3. M. Ren, R. Ravikrishna, and K. T. Valsaraj, Environ. Sci. Technol. 40, 7029 (2006). 
4. S.-D. Mo and W. Y. Ching, Phys. Rev. B 51, 13023 (1995).

5. A. L. Linsebigler, G. Lu, and J. T. J. Yates, Chem. Rev. 95, 735 (1995).

6. A. Mills and S. L. Hunte, J. Photochem. Photobiol. A Chem. 108, 1 (1997).

7. E. Yablonovitch, J. Mod. Opt. 41, 173 (1994).

8. K. Busch and S. John, Phys. Rev. E 58, 3896 (1998).

9. J. D. Joannopoulos, P. R. Villeneuve, and S. Fan, Nature 386, 143 (1997).

10. E. Yablonovitch, J. Opt. Soc. Am. B 10, 283 (1993)

11. Y. A. Vlasov, S. Petit, G. Klein, B. Hönerlage, and C. Hirlimann, Phys. Rev. E 60, 1030 (1999).

12. J. I. L. Chen, G. von Freymann, S. Y. Choi, V. Kitaev, and G. A. Ozin, J. Mater. Chem. 18, 369 (2008).

13. J. I. L. Chen, E. Loso, N. Ebrahim, and G. A. Ozin, J. Am. Chem. Soc. 130, 5420 (2008).

14. Y. Paz, Z. Luo, L. Rabenberg, and A. Heller, J. Mater. Res. 10, 2842 (1995)
15. M. J. Powell, R. G. Palgrave, C. W. Dunnill, and I. P. Parkin, Thin Solid Films 562, 223 (2014).

16. A. Mills and J. Wang, J. Photochem. Photobiol. A Chem. 182, 181 (2006).

17. C. A. Lane, D. E. Burton, and C. C. Crabb, Am. Chem. Soc. 61, 815 (1984).

18. I. Sopyan, M. Watanabe, S. Murasawa, K. Hashimoto, and A. Fujishima, J. Photochem. Photobiol. A Chem. 98, 79 (1996).

19. N. P. Mellott, C. Durucan, C. G. Pantano, and M. Guglielmi, Thin Solid Films 502, 112 (2006).

20. P. Sawunyama, A. Fujishima, and K. Hashimoto, Langmuir 15, 3551 (1999).

21. T. Minabe, et al., J. Photochem. Photobiol. A-Chemistry 137, 53 (2000).

22. Y. Ohko and A. Fujishima, J. Phys. Chem. 102, 1724 (1998).

23. N. Serpone and A. Emeline, Int. J. Photoenergy 4, 91 (2002).

24. J. I. L. Chen, Amplified Photochemistry with Slow Photons (2009).

25. O. Carp, C. L. Huisman, and A. Reller, Prog. Solid State Chem. 32, 33 (2004). 